\title{
Use of community-based subcutaneous furosemide in advanced heart failure: experience of a new service in the north-east of England
}

\author{
Authors: Andrew Brown, Judith Robson, Leonie Armstrong, Iain Matthews, Craig Runnett and Honey Thomas
}

\begin{abstract}
Aims
Evaluation of a community-based subcutaneous furosemide service for the treatment of advanced heart failure.
\end{abstract}

\section{Methods \\ We have initiated a community-based subcutaneous furosemide service for the treatment of advanced heart failure. In patients with end-stage heart failure, there is some evidence which suggests that furosemide can be given subcutaneously to relieve symptoms and avoid hospital admission. Our treatment guidelines were developed in collaboration with cardiologists, specialist nurses, palliative care and community nursing teams. All patients have a comprehensive emergency healthcare plan (EHCP) involving the heart failure team and delivery of the service is facilitated by community heart failure and district nurses in patients' homes. Our service aims to increase patient choice, reduce avoidable hospital admissions, facilitate early discharge and improve quality of life. We collected background and episode-related data for all patients with advanced heart failure that received subcutaneous furosemide.}

\section{Results}

Subcutaneous furosemide was used in 19 treatment episodes in 16 patients. The majority had left ventricular systolic dysfunction (LVSD) $(87 \%, 14 / 16)$. The median (interquartile range (IQR)) length of treatment was 10 (5-19) days. Median weight loss of 5 (2-7.5) $\mathrm{kg}$ was recorded over the course of treatment. Eleven patients' EHCPs were initially drawn up in secondary care and five in the community.

$50 \%(8 / 16)$ of patients died during treatment, $6 / 8$ died at home and 2/8 died at our specialist palliative care unit. Both patients were admitted as a result of difficulty managing the syringe driver at home. Of the treatment episodes which the patient survived, $18 \%(2 / 11)$ of patient episodes resulted in readmission. This was for assistance with syringe driver management and assessment following a fall at home.

Authors: Northumbria Healthcare NHS Trust, Newcastle upon Tyne, UK
For those patients who were alive on completion of initial subcutaneous furosemide course $(n=8)$ there were 10 admissions to hospital over a median survival period of 6 months. In the preceding 6 months there were 21 admissions in the same patient group.

\section{Conclusion}

Our data suggests that subcutaneous furosemide can be successfully delivered in the community. In addition to palliation in the final days of life, community subcutaneous furosemide can be an effective treatment leading to significant weight loss and improved symptoms and survival for several months. This approach allows management of worsening symptoms in the community and we have shown a reduction in hospital admissions in a group at high risk of hospital readmissions.

\section{Conflict of interest statement}

The authors have no conflict of interest to declare. 